Research Article

\title{
Group Invariant Solutions for Flow and Heat Transfer of Power-Law Nanofluid in a Porous Medium
}

\author{
Saba Javaid $\mathbb{D D}^{1}$ and Asim Aziz $\mathbb{D D}^{2}$ \\ ${ }^{1}$ School Natural Sciences, National University of Sciences and Technology, Sector H-12, Islamabad 45000, Pakistan \\ ${ }^{2}$ College of Electrical and Mechanical Engineering, National University of Sciences and Technology, Rawalpindi 46070, Pakistan
}

Correspondence should be addressed to Saba Javaid; saba_478@hotmail.com

Received 18 March 2021; Revised 10 May 2021; Accepted 18 May 2021; Published 25 May 2021

Academic Editor: A. M. Bastos Pereira

Copyright (c) 2021 Saba Javaid and Asim Aziz. This is an open access article distributed under the Creative Commons Attribution License, which permits unrestricted use, distribution, and reproduction in any medium, provided the original work is properly cited.

\begin{abstract}
The present work covers the flow and heat transfer model for the power-law nanofluid in the presence of a porous medium over the penetrable plate. The flow is caused by the impulsive movement of the plate embedded in Darcy's type porous medium. The flow and heat transfer model has been examined with the effect of linear thermal radiation and the internal heat source or sink in the flow regime. The Rosseland approximation is utilized for the optically thick nanofluid. To form the closed-form solutions for the governing partial differential equations of conservation of mass, momentum, and energy, the Lie symmetry analysis is used to get the reductions of governing equations and to find the group invariants. These invariants are then utilized to obtain the exact solution for all three cases, i.e., shear thinning fluid, Newtonian fluid, and shear thickening fluid. In the end, all solutions are plotted for the cu-water nanofluid and discussed briefly for the different emerging flow and heat transfer parameters.
\end{abstract}

\section{Introduction}

Nanotechnology has gained valuable attention since the concept of dispersing nanosized particles into the fluid stream was introduced. The improvements made in the thermal properties of ordinary fluids by adding the nanoscaled particles give a new direction for the study of heat transfer processes. Several studies reported that the thermal performance of nanofluids depends on the dispersion, stability, size, shape, and type of nanoparticles present in the system. The demand for new thermal devices in the electronics, automotive industries, heat exchangers, cooling devices, solar collectors, and many more leads to extensive research carried out to explain the processes of heat transfer in nanofluids [1-4]. The nanofluid flow and heat transfer over the flat surface had some interesting applications in industrial setups. The survey articles of Eastman [5], Wang et al. [6], Keblinski et al. [7], Buongiorno [8], Das et al. [9], Mahanthesh and Mackolil [10], Vanaki et al. [11], and the references therein cover significant literature on the nanofluid's flow over the flat surface.
In the aforementioned literature, nanofluids are often considered as the Newtonian fluids. Recently, researchers believe non-Newtonian fluid models are more appropriate for the analysis of nanofluids. This assumption is in line with the overall molecular behavior/characteristics of nanofluids. The comprehensive literature is available on the studies related to non-Newtonian nanofluid flow and heat transfer models. Madhu et al. [12] studied the effects of convective radiation for the second law in Carreau fluid flow. The author applied the numerical technique for the solution of the model and found the results for the various emerging parameters for flow and heat transfer. The nonsimilar modeling for mixed convection in magnetized second-grade nanofluid flow is given by Raees et al. [13]. The BVP4C solver is used to show that the graphical results and inferences are drawn for important quantities that are substantially affected by the physical parameters.

The power-law model is one of the most comprehensible non-Newtonian models as it covers a broad range of fluid properties. It is beyond the scope of this work to discuss vast literature on flow and heat transfer of power-law nanofluid 
under different thermophysical conditions. Some significant studies on power-law nanofluids flow and heat transfer are mentioned here. Santra et al. [14] investigated power-law nanofluid flow along with heat transfer. They consider water-based nanofluid including cu nanoparticles. The flow takes place within the rectangular channel, and the numerical solutions are computed for the modeled partial differential equations using the finite volume method. The results show that the addition of nanoparticles increases the heat transfer rate. Khana and Golra [15] present similar solutions of heat and mass transfer model for power-law nanofluid over a moving flat surface. An experiment is setup by Hojjat et al. [16] to study the impact of uniform distribution of nanoparticles in the ordinary fluid. They observed that the rheological behavior of nanofluids varies with temperature variations and an increase in the concentration of nanoparticles. Deng [17] and Deng et al. [18] presented the study of power-law nanofluid in a rectangular microchannel. Their results show that the heat transfer rate of electroosmotic flow is enhanced for shear thickening nanofluids. Aziz and Jamshed [19] reported heat transfer analysis for the MHD power-law nanofluid slip flow over a moving surface with variable thermal conductivity. Readers are recommended to study the latest research [20-28] on power-law nanofluids for further understanding.

Thermal radiation and heat source/sink effects are very important in the interpretation of many physical problems. The collected work for the non-Newtonian nanofluid with thermal radiation and heat source is available. Venkatesan and Reddy [29] studied the impact of the pulsating flow of $\mathrm{Al}_{2} \mathrm{O}_{3}$-blood non-Newtonian nanofluid in a channel in the presence of the magnetic field and thermal radiation. The effects of Joule heating and heat source are taken into account. The perturbation is applied to observe the effects of flow variables. Ramzan et al. [30] studied the effects of Newtonian heating and Fourier and Fick's laws on MHD dusty Casson fluid with variable heat source/sink. The numerical solutions are obtained via a BVP4C solver, and graphical results are also discussed for novel physical parameters. Some new research articles available in the literature elaborate on the effects of thermal radiation and heat source/sink on fluid velocity and temperature. See for example and the references therein [31-33].

Mathematical models involving non-Newtonian nanofluids are mostly solved using numerical and experimental techniques. The nonlinear nature of modeled differential equations made them difficult to solve analytically. The exact (closed-form) solutions are often preferred over numerical or experimental results to study the physical behavior of the fluid flow. Moreover, they are also used to determine the reliability of both computational and iterative approaches. There are few studies available in which exact solutions are sought for the nonlinear models. The study of MHD flow and radiative heat transfer characteristics of non-Newtonian nanofluids is reported by Maghsoudi et al. [34]. The author uses the Galerkin method to get the exact solution. The results show that the increasing magnetic force decreases the thermal efficiency and in turn decreases the skin friction coefficient and Nusselt number. The non-Newtonian nanofluid flow between two vertical plates is studied by Biswal et al. [35]. In this study, two methods are incurred to find the exact solutions, that is, Galerkin's method and the homotopy perturbation method. Aziz in his research found the exact solution of Stokes' flow of a non-Newtonian nanofluid in a porous medium by considering Navier's slip condition [36]. The author uses the Lie symmetry and generalized group approach to find the solutions. Tahiri and Mansouri [37] presented the analytical solutions using the Laplace-Ritz variational method for the flow of non-Newtonian nanofluid inside a circular tube. Aziz and Javaid [38] and Aziz et al. [39] applied the Lie symmetry method on the flow and heat transfer of MHD third grade nanofluid in the presence of thermal radiation and with uniform internal heat source/sink, respectively. The authors extract the exact closed-form solutions for governing partial differential equations. Kezzar et al. [40] established the study of natural convection of nonNewtonian nanofluid flow between two vertical flat plates. The exact solutions are obtained by using the Adomian generalized method (GDM), and numerical results are given by Runge-Kutta fourth-order. The symmetry analysis is a systematic way of calculating the self-similar solutions of nonlinear partial differential equations. Recently, some studies found are reported in literature in this context that are available in [41-47].

The literature survey reveals that no research reported exact solutions for the power-law nanofluid flow along with the process of heat transfer over the porous plate within the porous medium. In the present model, the flow is induced by the motion of the penetrable plate. The effects of Darcy's resistance and linear thermal radiation are incorporated in the flow model. The Tiwari and Das model [48] is used for the thermophysical properties of nanofluid. The exact solutions are found by using the Lie symmetry approach. Afterward, the solutions are plotted for cu-water nanofluid to observe the flow and heat transfer behavior of the fluid depending on the physical parameters. This prototype model is directly applicable in industrial processes such as glass and paper manufacturing, hot rolling and wire drawing, and thermal solar collectors. The final quality of products and the processes of heat transfer depend on the type of operating fluid and the stretching of bounding surfaces. Further applications of this mathematical model are in the fields of aerodynamic heating, crystal growing, and extraction of oil and gas.

\section{Model Statement}

Consider the unsteady unidirectional Stokes' flow of an incompressible fluid over an infinite penetrable plate in the presence of thermal radiation and uniform heat source/sink embedded in a porous medium. The fluid motion is caused by an arbitrary motion of the plate. The flow is assumed to be in the $x$-direction which is chosen along with the penetrable plate and $y$-axis is perpendicular to it. The velocity field for the flow is given by

$$
\bar{V}=\left[v(y, t),-v_{0}, 0\right] .
$$


In above equation, $v_{0}$ is the constant suction/injection velocity. $v_{0}<0$ specify the suction and $v_{0}>0$ represents the injection velocity. Considering the preceding assumptions, the governing boundary layer equations for the flow and heat transfer model are [49]

$$
\begin{gathered}
\frac{\partial v}{\partial x}-\frac{\partial v_{0}}{\partial y}=0 \\
\rho_{\mathrm{nf}}\left(\frac{\partial v}{\partial t}-v_{0} \frac{\partial v}{\partial y}\right)=\mu_{\mathrm{nf}} \frac{\partial \tau_{\mathrm{xy}}}{\partial y}+R_{x} \\
\left(\rho C_{P}\right)_{\mathrm{nf}}\left(\frac{\partial T}{\partial t}-v_{0} \frac{\partial T}{\partial y}\right)=\frac{\partial}{\partial y}\left(\kappa_{\mathrm{nf}} \frac{\partial T}{\partial y}\right)-\frac{\partial q_{r}}{\partial y}-Q\left(T-T_{\infty}\right) .
\end{gathered}
$$

It can be noticed that equation (2) is trivially satisfied for the given velocity field. In equations (2)-(4), $\rho_{\mathrm{nf}}$ is the nanofluid density, $\tau_{x y}$ is the stress tensor, $R_{x}$ is Darcy's resistance, $C_{\mathrm{Pnf}}$ is the specific heat capacity, $\kappa_{\mathrm{nf}}$ is the thermal conductivity, $q_{r}$ is the thermal radiation, and $Q$ is the internal heat source/sink. The internal heat generation is represented by $Q>0$, whereas $Q<0$ refers the internal heat absorption. The correlations for thermophysical properties of nanofluid are given in Table 1 [50-53].

In Table $1, \varphi$ is the volume concentration of nanoparticles. The parameters $\rho_{f}, \mu_{f}, \sigma_{f},\left(\rho C_{P}\right)_{f}$, and $\kappa_{f}$ are density, viscosity, electrical conductivity, specific heat capacity, and thermal conductivity of base fluid, respectively. Quantities $\rho_{s}, \mu_{s}, \sigma_{s},\left(\rho C_{P}\right)_{s}$, and $\kappa_{s}$ are density, viscosity, electrical conductivity, specific heat capacity, and thermal conductivity of nanoparticles.

The expression for stress tensor in equations (3) is given by [54]

$$
\tau_{x y}=K\left|\frac{\partial v}{\partial y}\right|^{m-1} \frac{\partial v}{\partial y}
$$

where $K$ is the coefficient of consistency and $m$ is the characteristic coefficient for the non-Newtonian behavior of the fluid. The relation between the pressure gradient and the velocity for the unidirectional flow of power-law nanofluids is calculated as

$$
R_{x}=-\frac{\phi}{\kappa}\left(K\left|\frac{\partial v}{\partial y}\right|^{m-1}\right) v,
$$

where $\phi$ is the porosity and $\kappa$ is the permeability of the porous medium. The radiation term in equation (4) under Rosseland approximations $[55,56]$ is simplified to

$$
\frac{\partial q_{r}}{\partial y}=-\frac{16 \sigma^{*} T_{\infty}^{3}}{3 \kappa^{*}} \frac{\partial^{2} T}{\partial y^{2}} .
$$

In above expression, $\sigma^{*}$ and $\kappa^{*}$ are the Stefan-Boltzmann constant and the mean absorption coefficient, respectively. $T_{\infty}$ is the ambient temperature. By employing the above simplified expressions given in equations (6) and (7), the momentum equation (3) and the energy equation (4) can be written as

$$
\begin{aligned}
& \rho_{\mathrm{nf}}\left(\frac{\partial v}{\partial t}-v_{0} \frac{\partial v}{\partial y}\right)=\mu_{\mathrm{nf}} \frac{\partial}{\partial y}\left(K\left|\frac{\partial v}{\partial y}\right|^{m-1} \frac{\partial^{2} v}{\partial y^{2}}\right)-\frac{\phi}{\kappa}\left(K\left|\frac{\partial v}{\partial y}\right|^{m-1}\right) v, \\
&\left(\rho C_{P}\right)_{\mathrm{nf}}\left(\frac{\partial T}{\partial t}-v_{0} \frac{\partial T}{\partial y}\right)= \frac{\partial}{\partial y}\left(\kappa_{\mathrm{nf}} \frac{\partial T}{\partial y}\right) \\
&+\frac{16 \sigma^{*} T_{\infty}^{3}}{3 \kappa^{*}} \frac{\partial^{2} T}{\partial y^{2}}-Q\left(T-T_{\infty}\right) .
\end{aligned}
$$

The respective initial and boundary conditions are

$$
\begin{array}{rr}
v(0, t)=V_{0} U(t), T(0, t)=h(t)+T_{\infty}, & t>0, \\
v(y, t) \longrightarrow 0, T(y, t) \longrightarrow T_{\infty}, & \text { as } y \longrightarrow \infty, t>0, \\
v(y, 0)=f(y), T(y, 0)=g(y)+T_{\infty}, & y>0,
\end{array}
$$

where $V_{0}$ is the reference velocity, and $U(t), h(t), f(y)$, and $g(y)$ are the unknown functions.

To make equations (8)-(10) dimensionless, we introduce nondimensional parameters as

$$
\begin{aligned}
v & =V_{0} \bar{v} \\
t & =\frac{L}{V_{0}} \bar{t} \\
y & =L\left(\frac{K}{\rho_{f} V_{0}^{2-m} L^{m}}\right)^{1 /(m+1)} \bar{y} \\
v_{0} & =V_{0}\left(\frac{K}{\rho_{f} V_{0}^{2-m} L^{m}}\right)^{1 /(m+1)} \bar{v}_{0}, \\
\bar{\tau}_{\overline{\mathrm{xy}}} & =\left|\frac{\partial v}{\partial y}\right|^{m-1} \frac{\partial v}{\partial y}=\left(\frac{K V_{0}^{3-m} \rho_{f}}{L^{m}}\right)^{-1 /(m+1)} \tau_{\mathrm{xy}}, \\
\frac{\phi}{\kappa} & =\bar{\phi}\left(\frac{\rho_{f} V_{0}^{2-m} L^{m}}{K}\right)^{2 /(m+1)} \frac{1}{L^{2}}, \\
\bar{\theta} & =\frac{T-T_{\infty}}{T_{w}-T_{\infty}} .
\end{aligned}
$$

After neglecting bars and the substitution of dimensionless parameters in equations (8) and (9), the nondimensional forms of momentum and energy equation are

$$
\begin{aligned}
& \frac{\partial v}{\partial t}-v_{0} \frac{\partial v}{\partial y}=\left|\frac{\partial v}{\partial y}\right|^{m-1}\left(m \mu \frac{\partial v}{\partial y}-\psi v\right), \\
& \frac{\partial \theta}{\partial t}-v_{0} \frac{\partial \theta}{\partial y}=\left(\frac{\kappa_{*}+\mathrm{Nr}}{\operatorname{Pr}\left(\rho C_{P}\right)_{*}}\right) \frac{\partial^{2} \theta}{\partial y^{2}}+\Phi \theta .
\end{aligned}
$$

In equation (12), $\mu=1 /(1-\varphi)^{2.5} \varphi_{*}$ is the flow parameter and $\psi=\bar{\phi} / \varphi_{*}$ is the permeability parameter with $\varphi_{*}=$ $1-\varphi+\varphi\left(\rho_{s} / \rho_{f}\right)$. In equation (13), $\mathrm{Nr}=16 T^{3} \sigma^{*} / 3 \kappa_{f} \kappa^{*}$ is the radiation parameter, $\operatorname{Pr}=\left(V_{0}^{3(m-1) /(m+1)} L^{(1-m) /(1+m)}\right.$ 
TABLE 1: Correlations between physical properties of base fluid and nanoparticles.

\begin{tabular}{lc}
\hline Properties & Nanofluid \\
\hline Density $(\rho)$ & $\rho_{\mathrm{nf}}=\rho_{f}(1-\varphi)+\varphi \rho_{s}$ \\
Viscosity $(\mu)$ & $\mu_{\mathrm{nf}}=\mu_{f} /(1-\varphi)^{2.5}$ \\
Electrical conductivity $(\sigma)$ & $\left.\left.\left.\sigma_{\mathrm{nf}}=\sigma_{f}\left[1+\left(3\left(\sigma_{s} / \sigma_{f}\right)-1\right) \varphi\right) /\left(\sigma_{s} / \sigma_{f}\right)+2-\left(\left(\sigma_{s} / \sigma_{f}\right)-1\right) \varphi\right)\right)\right]$ \\
Heat capacity $\left(\rho C_{P}\right)$ & $\left(\rho C_{P}\right)_{\mathrm{nf}}=\left(\rho C_{P}\right)_{f}\left[(1-\varphi)+\varphi\left(\left(\rho C_{P}\right) /\left(\rho C_{P}\right)_{f}\right)\right]$ \\
Thermal conductivity $(\kappa)$ & $\kappa_{\mathrm{nf}}=\kappa_{f}\left[\left(\kappa s+2 \kappa_{f}-2 \varphi\left(\kappa_{f}-\kappa_{s}\right)\right) /\left(\kappa_{s}+2 \kappa_{f}+\varphi\left(\kappa_{f}-\kappa_{s}\right)\right)\right]$ \\
\hline
\end{tabular}

$\left.K^{2 /(m+1)} \rho_{f}^{(m /(m+1))\left(C_{P}\right)_{f}}\right) / \kappa_{f}$ is the Prandtl number, and $\Phi=$ $Q L / V_{0} \rho_{f}\left(C_{P}\right)_{f}$ is the heat source/sink parameter. For convenience, write $\left[\left(\kappa_{s}+2 \kappa_{f}-2 \varphi\left(\kappa_{f}-\kappa_{s}\right)\right) /\left(\kappa_{s}+2 \kappa_{f}+\varphi\left(\kappa_{f}-\right.\right.\right.$ $\left.\left.\left.\kappa_{s}\right)\right)\right]$ as $\kappa_{*}$ and $1-\varphi+\varphi\left(\left(\rho C_{P}\right)_{s} /\left(\rho C_{P}\right)_{f}\right)$ as $\left(\rho C_{P}\right)_{*}$. From equation (10), transformed boundary conditions are

$$
\begin{aligned}
& v(0, t)=U(t), \\
& \theta(0, t)=H(t), \\
& t>0, \\
& v(y, t) \longrightarrow 0, \\
& \theta(y, t) \longrightarrow 0, \\
& \text { as } y \longrightarrow \infty, t>0, \\
& v(y, 0)=F(y), \\
& \theta(y, 0)=G(y), \\
& y>0,
\end{aligned}
$$

where $H(t)=h(t) /\left(T_{w}-T_{\infty}\right)$ and $G(t)=g(t) /\left(T_{w}-T_{\infty}\right)$.

\section{Lie Symmetry Analysis}

This section covers the procedure adopted to find the Lie point symmetries for the momentum and energy equations given in (12) and (13) respectively. Symmetry transformations are then used to form the group invariant solutions. The aim is to find the symmetry operator of the form

$$
\Pi=\Gamma^{1}(t, y, u) \frac{\partial}{\partial t}+\Gamma^{2}(t, y, u) \frac{\partial}{\partial y}+\Gamma^{3}(t, y, u) \frac{\partial}{\partial v} .
$$

$\Pi$ is the symmetry generator of second-order PDE if and only if

$$
\left.\Pi^{[2]}[\mathrm{PDE}]\right|_{\mathrm{PDE}=0}=0 \text {, }
$$

where $\Pi^{[2]}$ is the second prolonged operator of $\Pi$, that can be written as

$$
\Pi^{[2]}=\Pi+\mathrm{F}^{t} \frac{\partial}{\partial v_{t}}+\mathrm{F}^{y} \frac{\partial}{\partial v_{y}}+\mathrm{F}^{y y} \frac{\partial}{\partial v_{y y}},
$$

together with

$$
\begin{gathered}
\mathrm{F}^{t}=D_{t} \Gamma_{3}-v_{t} D_{t} \Gamma_{1}-v_{y} D_{t} \Gamma_{2}, \\
\mathrm{~F}^{y}=D_{y} \Gamma_{3}-v_{t} D_{y} \Gamma_{1}-v_{y} D_{y} \Gamma_{2}, \\
\mathrm{~F}^{y y}=D_{y} \mathrm{~F}^{y}-v_{t y} D_{y} \Gamma_{1}-v_{y y} D_{y} \Gamma_{2} .
\end{gathered}
$$

The total derivatives operators are given by

$$
\begin{gathered}
D_{t}=\partial_{t}+v_{t} \partial_{v}+v_{t t} \partial_{v_{t}}+v_{t y} \partial_{v_{y}}+\cdots \\
D_{y}=\partial_{y}+v_{t} \partial_{v}+v_{y y} \partial_{v_{y}}+v_{t y} \partial_{v_{t}}+\cdots
\end{gathered}
$$

3.1. Lie Symmetry Analysis of the Flow Model. In this subsection, Lie symmetry analysis is applied to the flow equation (12). The equation (18) along with equations (12) and (19)-(22) reduces to the following overdetermined system of partial differential equations

$$
\begin{aligned}
\Gamma_{y}^{1}=\Gamma_{y}^{3}=\Gamma_{v}^{1}=\Gamma_{t}^{3}=\Gamma_{v}^{2} & =0, \\
\Gamma_{t}^{2}-v_{0}\left(\Gamma_{y}^{2}-\Gamma_{t}^{1}+v_{0} \Gamma_{y}^{1}\right) & =0, \\
\Gamma^{3}+(m-2) v \psi \Gamma_{v}^{3}+v \psi \Gamma_{t}^{1}+v \psi \Gamma_{y}^{2}-m v \psi \Gamma_{y}^{2} & =0, \\
(m-1) m \mu \Gamma_{v}^{3}+(m-2) v \psi \Gamma_{v}^{2}+m \mu \Gamma_{t}^{1}-m^{2} \mu \Gamma_{y}^{2} & =0 .
\end{aligned}
$$

The solution of above system gives rise to two cases with respect to the power-law index $m$ and presents a three-dimensional Lie algebra.

Case I. When $\mu \neq 0, \psi \neq 0$, and $m=2$,

$$
\begin{aligned}
& \Pi_{1}=\frac{\partial}{\partial t}, \\
& \Pi_{2}=\frac{\partial}{\partial y}, \\
& \Pi_{3}=t \frac{\partial}{\partial t}-t v_{0} \frac{\partial}{\partial y}-v \frac{\partial}{\partial v} .
\end{aligned}
$$

Case II. When $\mu \neq 0, m(m-1)(m-2) \neq 0, \psi \neq 0$,

$$
\begin{aligned}
& \Pi_{1}=\frac{\partial}{\partial t}, \\
& \Pi_{2}=\frac{\partial}{\partial y} \\
& \Pi_{3}=(1-m) t \frac{\partial}{\partial t}+t v_{0}(m-1) \frac{\partial}{\partial y}+v \frac{\partial}{\partial v}
\end{aligned}
$$




\section{Exact Solution for Pseudoplastic Fluids $(m>1)$}

In this section, the exact solution is derived for the flow of nanofluid in the case $m=2$. This gives the physical interpretation of pseudoplastic (shear thickening) fluids. The momentum equation (12) for $m=2$ can be written as

$$
\frac{\partial v}{\partial t}-v_{0} \frac{\partial v}{\partial y}=\left(\frac{\partial v}{\partial y}\right)\left(2 \mu \frac{\partial^{2} v}{\partial y^{2}}-\psi v\right) .
$$

The symmetry operators given in Case I are considered to solve the PDE (26).

4.1. Group Invariant Solution Corresponding to $\Pi_{1}+c \Pi_{2}$. The invariant solution corresponding to the linear combination of time and space translation generators $\Pi_{1}+c \Pi_{2}$ with $(c>0)$ represents a forward travelling wave solution. After solving the characteristic equation, the invariant is given by

$$
v(y, t)=G(\eta), \quad \text { where } \eta=y-c t .
$$

Using the relation written in equation (27), the PDE (26) reduces to the first-order ordinary differential equation

$$
-c \frac{\partial G}{\partial \eta}-v_{0} \frac{\partial G}{\partial \eta}-\frac{\partial G}{\partial \eta}\left(2 \mu \frac{\partial^{2} G}{\partial \eta^{2}}-\psi G(\eta)\right)=0,
$$

along with the initial condition as

$$
G(0)=1 \text {. }
$$

The solution of equation (28) subjected to the initial condition (29) gives the exact solution for $G(\eta)$ as

$$
G(\eta)=\exp \left(-\sqrt{\frac{\psi}{2 \mu} \eta}\right)
$$

Substituting $G(\eta)$ from equation (30) into equation (27), the group invariant solution will be

$$
v(y, t)=\exp \left(-\sqrt{\frac{\psi}{2 \mu}}(y-c t)\right),
$$

provided that the following compatibility condition holds

$$
c=-v_{0} \text {. }
$$

The solution given in equation (31) satisfies the initial and boundary conditions (14)-(16) with

$$
\begin{aligned}
& U(t)=\exp \left(\sqrt{\frac{\psi}{2 \mu}} c t\right), \\
& F(y)=\exp \left(-\sqrt{\frac{\psi}{2 \mu}} y\right) .
\end{aligned}
$$

It can be noticed from the above equation that $U(t)$ and $F(y)$ are depending on the flow and permeability parameter.
4.2. Results and Discussion. In this subsection, the solution (31) is plotted against different governing parameters that elaborate the effect on fluid velocity. Graphs are produced for the water-based cu-water nanofluid. Figure 1(a) shows the effect of nanoparticle concentration on the velocity of shear thickening fluid. The ratio $\varphi$ controls the viscosity and the thermal conductivity of the nanofluid, such that the linear velocity of the flow decreases by increasing the nanoparticle concentration. The effect of permeability parameter on fluid velocity is shown in Figure 1(b). The presence of a porous medium causes higher resistance to the fluid flow due to which the fluid decelerates. This causes thinning of the momentum boundary layer. The velocity of the nanofluid gradually decreases to free stream velocity as shown in Figure 1(c). The suction effect, that is, $v_{0}>0$ is depicted in Figure 1(d). The fluid velocity decreases for increasing values of $v_{0}$ through penetrable surface, whereas the opposite behavior is observed for the case of injection $v_{0}<0$

\section{Exact Solution for Newtonian Fluids $(m=1)$}

The momentum equation (12) for the Newtonian fluid $(m=$ 1) converts into

$$
\frac{\partial v}{\partial t}-v_{0} \frac{\partial v}{\partial y}=\left(\mu \frac{\partial^{2} v}{\partial y^{2}}-\psi v\right)
$$

where the exact solution of equation (34) will be formed corresponding to symmetry generators given in Case II.

5.1. Group Invariant Solution Corresponding to $\Pi_{1}-c \Pi_{2}$. The backward type travelling wave solution can be found by the linear combination of time and space translation generators $\Pi_{1}-c \Pi_{2}$. Here, $c>0$ gives the speed of the waves. In this case, the waves are propagated towards the plate. The invariant solution is of the form

$$
v(y, t)=Z(\xi), \quad \text { where } \xi=y+c t
$$

Substitution of above expression in equation (34) reduces it to the first-order ODE

$$
c \frac{\mathrm{d} Z}{\mathrm{~d} \xi}-v_{0} \frac{\mathrm{d} Z}{\mathrm{~d} \xi}=\mu \frac{\mathrm{d}^{2} Z}{\mathrm{~d} \xi^{2}}-\psi Z(\xi),
$$

along with the initial condition

$$
Z(0)=1
$$

The exact solution of equation (36) with respect to equation (37) is

$$
Z(\xi)=\exp \left(\frac{\left(c-v_{0}\right)-\sqrt{\left(c-v_{0}\right)^{2}+4 \psi \mu}}{2 \mu} \xi\right)
$$

Equation (35) together with equation (38) takes the form 

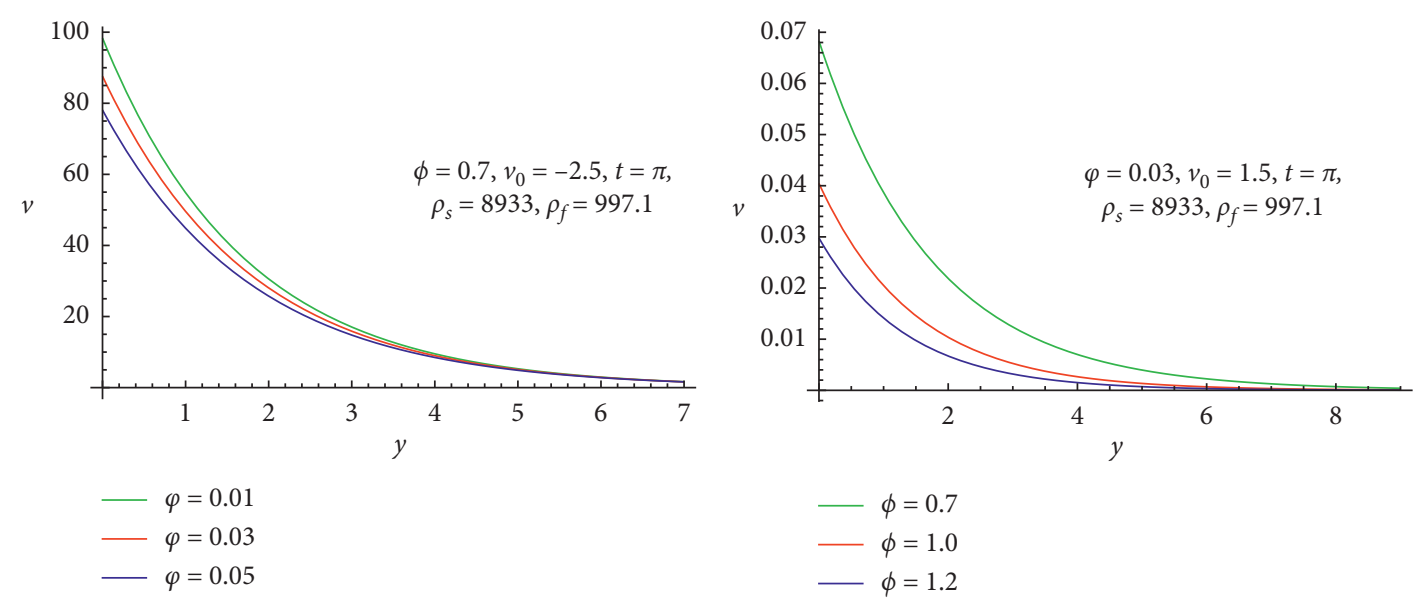

(a)

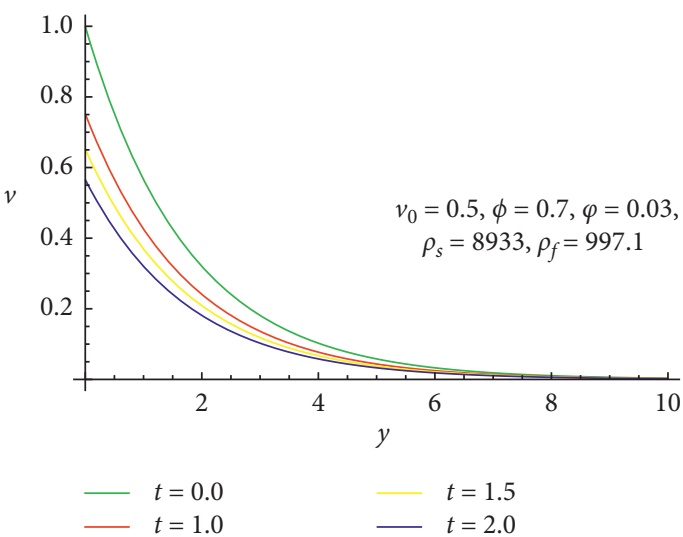

(c)

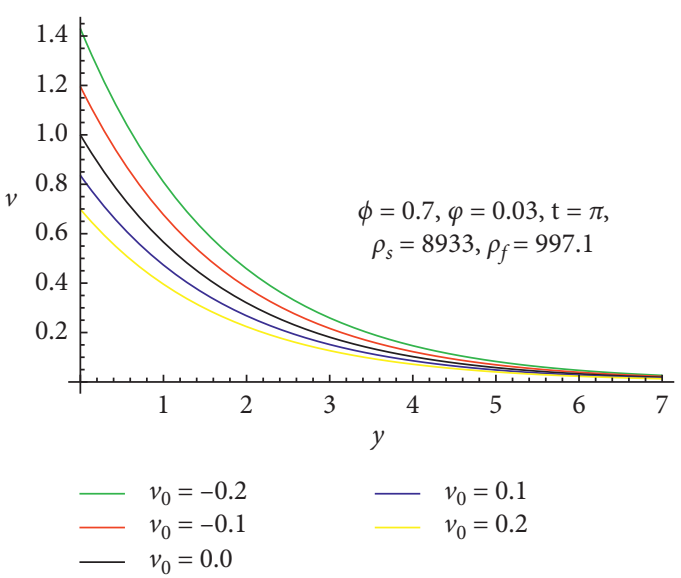

(d)

Figure 1: Velocity profile (31) for various values of $\varphi, \phi, t$, and $v_{0}$.

$$
v(y, t)=\exp \left(\frac{\left(c-v_{0}\right)-\sqrt{\left(c-v_{0}\right)^{2}+4 \psi \mu}}{2 \mu}(y+c t)\right) .
$$

The above solution satisfies the boundary condition when

$$
\begin{aligned}
& U(t)=\exp \left(\frac{\left(c-v_{0}\right)-\sqrt{\left(c-v_{0}\right)^{2}+4 \psi \mu}}{2 \mu} c t\right), \\
& F(y)=\exp \left(\frac{\left(c-v_{0}\right)-\sqrt{\left(c-v_{0}\right)^{2}+4 \psi \mu}}{2 \mu} y\right) .
\end{aligned}
$$

5.2. Results and Discussion. The effect of different physical parameters on velocity profile (39) is shown in Figure 2. Figure 2(a) shows the effect of increasing nanoparticles volume concentration on the nanofluid velocity. The velocity of the Newtonian fluid within the boundary layer region increases with the addition of nanoparticles. The increasing permeability of the porous medium shows the decrease in a fluid motion. The number of pores causes more resistance within the fluid (Figure 2(b)). In Figure 2(c), the overall effect of suction $v_{0}>0$ and injection $v_{0}<0$ is presented. The fluid velocity for the case of suction $v_{0}>0$ shows the increasing behavior, whereas the opposite trend is observed in the case of injection. Moreover, the fluid velocity continues to decrease with time as depicted in Figure 2(d). Finally, the ascending wave speed slows down fluid motion within the boundary layer, as shown in Figure 2(e).

\section{Exact Solution for Dilatant Fluid $(m<1)$}

In Case II, if we take $m=-2$, this will represent the shear thinning fluids, and the symmetry operators in this case takes the form 


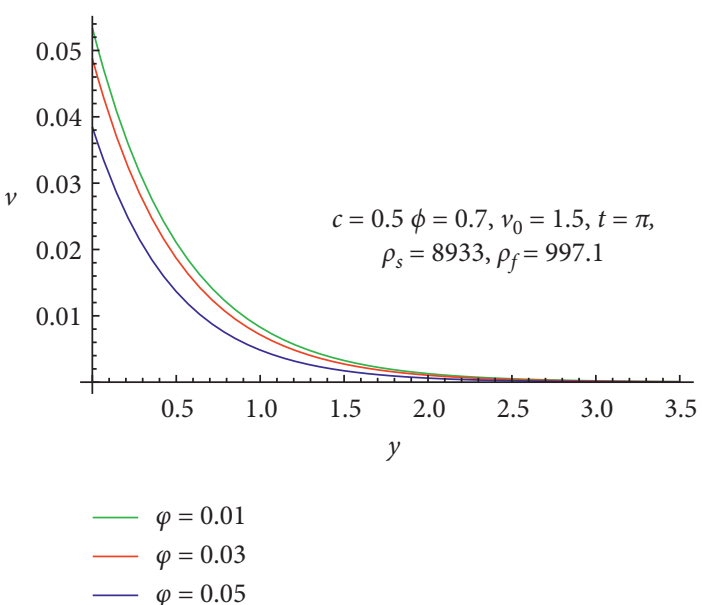

(a)

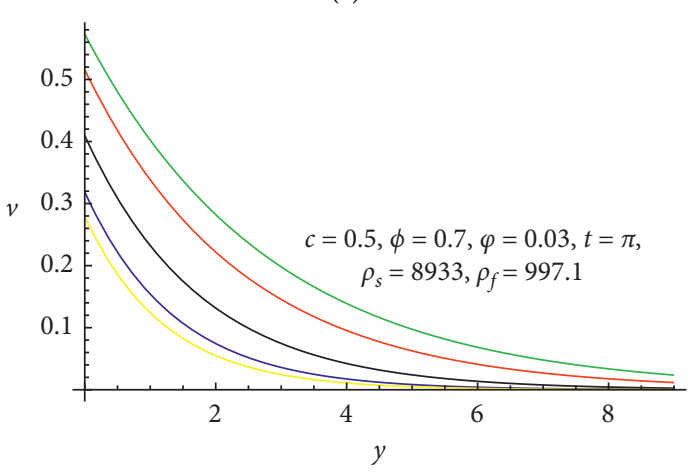

(c)

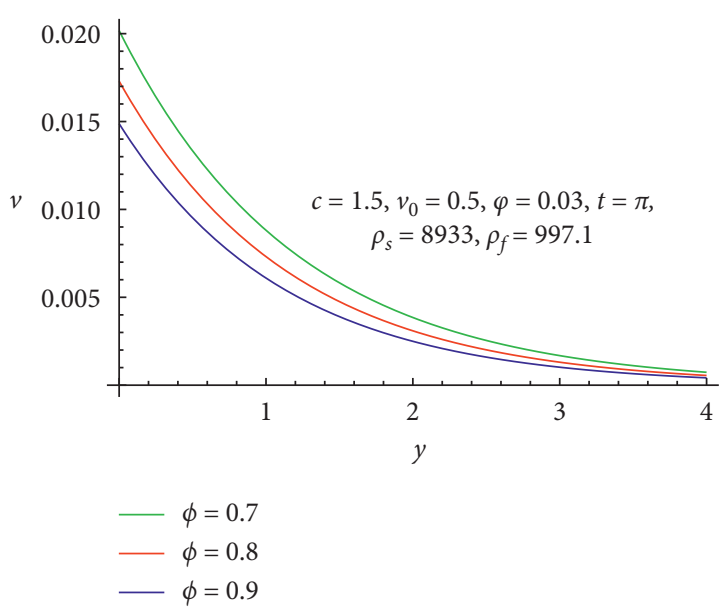

(b)

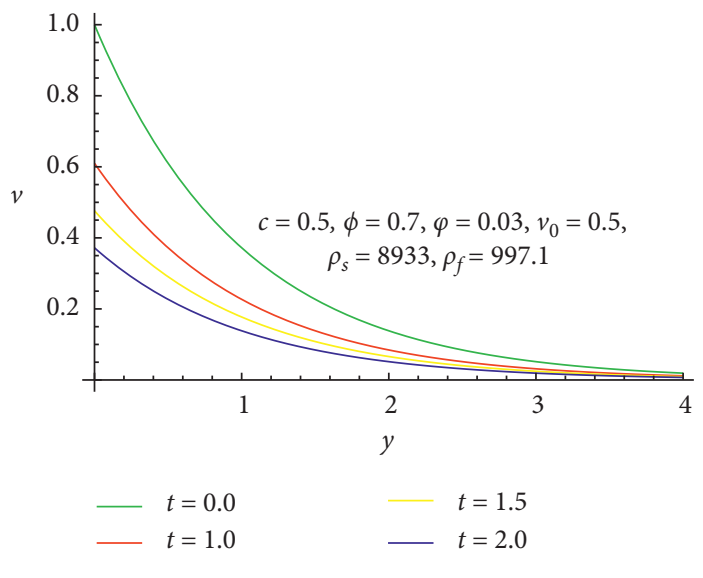

(d)

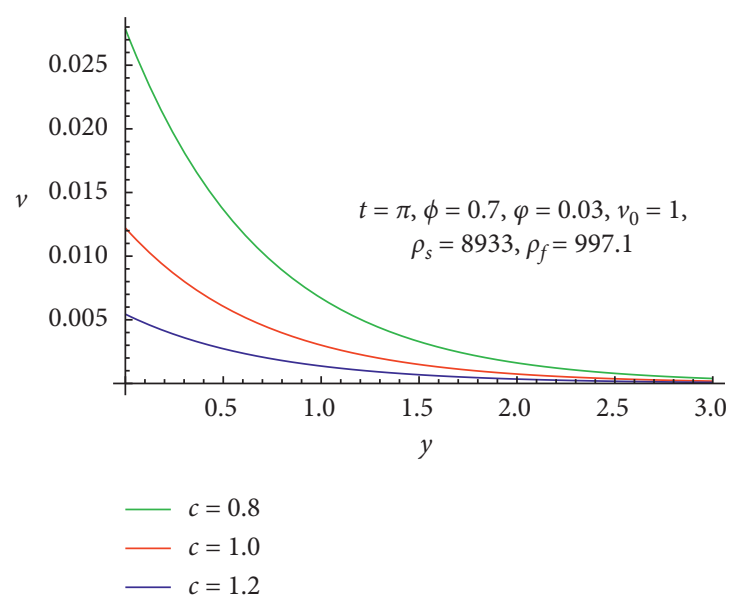

(e)

FigURE 2: Velocity profile (39) for various values of $\varphi, \phi, v_{0}, t$, and $c$. 


$$
\begin{aligned}
& \Pi_{1}=\frac{\partial}{\partial t}, \\
& \Pi_{2}=\frac{\partial}{\partial y}, \\
& \Pi_{3}=3 t \frac{\partial}{\partial t}-3 t v_{0} \frac{\partial}{\partial y}+v \frac{\partial}{\partial v} .
\end{aligned}
$$

The momentum equation (12) for $m=-2$ can be written as

$$
\frac{\partial v}{\partial t}-v_{0} \frac{\partial v}{\partial y}=\left(\frac{\partial v}{\partial y}\right)^{-3}\left(-2 \mu \frac{\partial^{2} v}{\partial y^{2}}-\psi v\right) .
$$

The exact solution will be form corresponding to above three-dimensional Lie algebra.

6.1. Group Invariant Solution Corresponding to $\Pi_{1}+c \Pi_{2}$. Consider the symmetry generators with the linear combination of time and space translation operators. This type of combination represents the backward-type travelling wave solution. The invariants are assumed as

$$
v(y, t)=R(\delta), \quad \text { where } \delta=y-c t .
$$

The above relation together with equation (12) for $m=$ -2 gives the reduced first-order ODE as

$$
\left(-c-v_{0}\right)\left(\frac{\mathrm{d} R}{\mathrm{~d} \delta}\right)^{4}+2 \mu \frac{\mathrm{d}^{2} R}{\mathrm{~d} \delta^{2}}+\psi R(\delta)=0 .
$$

The initial condition is

$$
R(\delta)=1, \quad \text { for } \delta=0 .
$$

Considering the generalized compatibility approach given in [57], the general solution has the form

$$
R(\delta)=A \exp (B \delta)
$$

where parameters $A$ and $B$ are to be determined. Using equation (46) into equation (44) admits the exact solution of the form

$$
R(\delta)=\exp \left(-\sqrt{\frac{\psi}{2 \mu}} \delta\right) .
$$

Substitution of above expression into equation (43) gives the exact solution of the momentum equation (12) for the dilatant fluids. That is,

$$
v(y, t)=\exp \left(-\sqrt{\frac{\psi}{2 \mu}}(y-c t)\right),
$$

where the speed of travelling wave $c=-v_{0}$. The above solution satisfies the boundary conditions with

$$
\begin{aligned}
& U(t)=\exp \left(\sqrt{\frac{\psi}{2 \mu}} c t\right), \\
& F(y)=\exp \left(-\sqrt{\frac{\psi}{2 \mu}} y\right) .
\end{aligned}
$$

6.2. Results and Discussion. The solution (48) is plotted for the various emerging parameters in Figure 3. The qualitative behavior of dilatant nanofluids is the same as in case of pseudoplastic fluids.

\section{Lie Symmetry Analysis of Energy Equation}

The Lie symmetry analysis of energy equation (13) is presented in this section. In equation (18), substitute equation (13) in the place of PDE along with equations (19)-(22). This will generate a system

$$
\begin{aligned}
\Gamma_{y}^{1} & =\Gamma_{\theta}^{2}=\Gamma_{\theta}^{1}=0, \\
& \Gamma_{\theta \theta}^{3}-\Phi \theta \Gamma_{\theta}^{1}-2\left(\Gamma_{y \theta}^{2}-v_{0} \Gamma_{y \theta}^{1}\right)=0, \\
& -\operatorname{Pr}\left(\rho C_{p}\right)_{*} \Phi \theta \Gamma_{\theta}^{1}-\Gamma_{t}^{1}-2 \Gamma_{y}^{2}-3 v_{0} \Gamma_{y}^{1}+\left(\mathrm{Nr}+\kappa_{*}\right) \Gamma_{\mathrm{yy}}^{1}=0, \\
& -\operatorname{Pr}\left(\rho C_{P}\right)_{*}\left(\Phi \Gamma^{3}-\Phi \theta \Gamma_{\theta}^{3}+\Phi^{2} \theta^{2} \Gamma_{\theta}^{1}-\Gamma_{t}^{3}+\Phi \theta \Gamma_{t}^{1}+v_{0} \Gamma_{y}^{3}-\Phi \theta v_{0} \Gamma_{y}^{1}\right) \\
& -\left(\mathrm{Nr}+\kappa_{*}\right)\left(\Gamma_{\mathrm{yy}}^{3}-\Phi \theta \Gamma_{\mathrm{yy}}^{1}\right)=0, \\
& -\operatorname{Pr}\left(\rho C_{P}\right)_{*}\left(\Phi \theta \Gamma_{\theta}^{2}+\Phi \theta v_{0} \Gamma_{\theta}^{1}+\Gamma_{t}^{2}+v_{0} \Gamma_{t}^{1}-v_{0} \Gamma_{y}^{2}-v_{0}^{2} \Gamma_{y}^{1}\right) \\
& -\left(\mathrm{Nr}+\kappa_{*}\right)\left(2 \Gamma_{y \theta}^{3}-2 \Phi \theta \Gamma_{y \theta}^{1}-\Gamma_{\mathrm{yy}}^{2}-v_{0} \Gamma_{\mathrm{yy}}^{1}\right)=0 .
\end{aligned}
$$



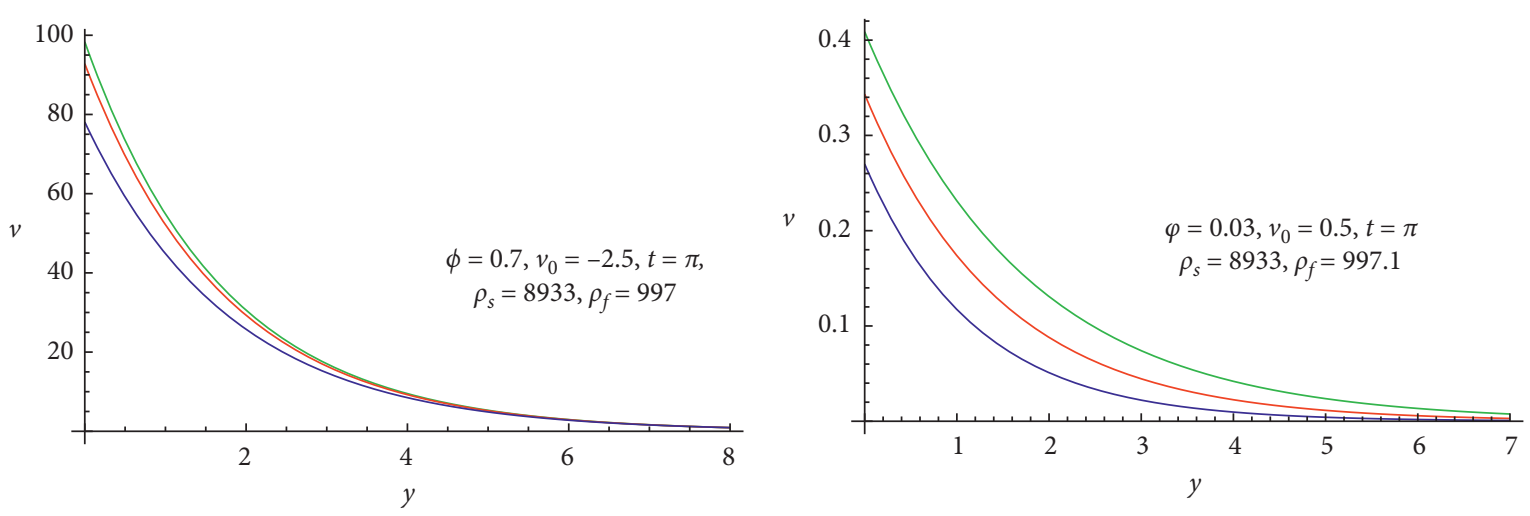

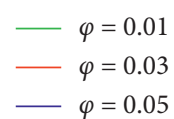

$-\varphi=0.05$

(a)

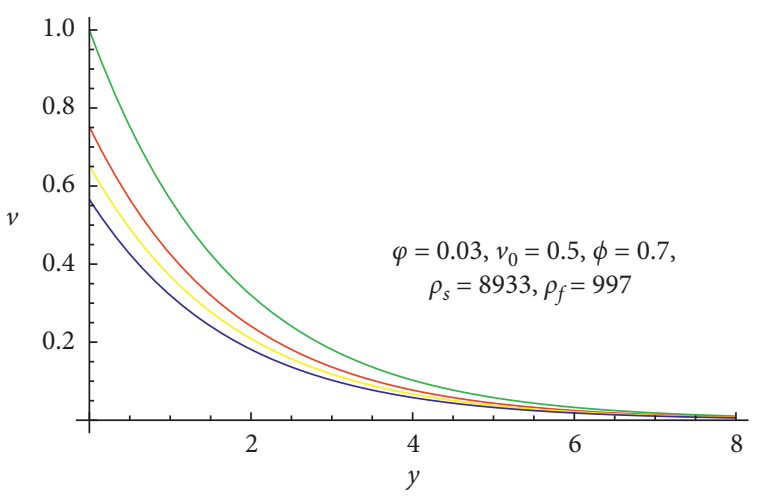

$\phi=0.7$

$\longrightarrow \phi=1.0$

$\longrightarrow \phi=1.5$

(b)

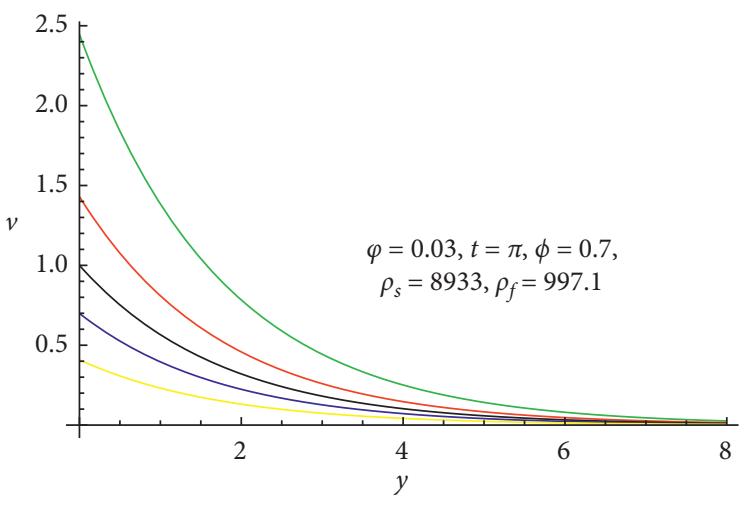

(d)

(c)

Figure 3: Velocity profile (48) for various values of $\varphi, \phi$, $t$, and $v_{0}$.

The solution of the above overdetermined system of linear partial differential equations gives the infinite-dimensional Lie algebra generated by

$$
\begin{aligned}
& \Pi_{1}=\frac{\partial}{\partial t}, \\
& \Pi_{2}=\frac{\partial}{\partial y}, \\
& \Pi_{3}=\frac{\partial}{\partial \theta}, \\
& \Pi_{4}=2 t \frac{\partial}{\partial y}+\left(\frac{-\operatorname{Pr}\left(\rho C_{P}\right)_{*} \theta\left(v_{0} t+y\right)}{\mathrm{Nr}+\kappa_{*}}\right) \frac{\partial}{\partial \theta^{\prime}} \\
& \Pi_{5}=4 t \frac{\partial}{\partial t}+2 y \frac{\partial}{\partial y}+\left(4 \Phi t \theta-\frac{\operatorname{Pr}\left(\rho C_{P}\right)_{*} v_{0}}{\mathrm{Nr}+\kappa_{*}}\left(t v_{0}-y\right) \theta\right) \frac{\partial}{\partial \theta}, \\
& \Pi_{6}=4 t^{2} \frac{\partial}{\partial t}+4 t y \frac{\partial}{\partial y}+\left(-2 t \theta+4 \Phi t^{2} \theta-\frac{\operatorname{Pr}\left(\rho C_{P}\right)_{*}}{\mathrm{Nr}+\kappa_{*}}\left(v_{0} t+y\right)^{2} \theta\right) \frac{\partial}{\partial \theta^{\prime}}, \\
& \Pi_{\infty}=\mathrm{F}(y, t) \frac{\partial}{\partial \theta},
\end{aligned}
$$


where $\mathrm{F}(y, t)$ satisfies the equation

$$
\operatorname{Pr} \Phi\left(\rho C_{P}\right)_{*} \mathrm{~F}(y, t)+\operatorname{Pr}\left(\rho C_{P}\right)_{*} \frac{\partial \mathrm{F}}{\partial t}-\operatorname{Pr} v_{0}\left(\rho C_{P}\right)_{*} \frac{\partial \mathrm{F}}{\partial y}-\left(\mathrm{Nr}+\kappa_{*}\right) \frac{\partial^{2} \mathrm{~F}}{\partial y^{2}}
$$

\section{Exact Solution of Energy Equation}

The symmetry operators given in equation (51) are utilized to find the group invariant solution of equation (13).

8.1. Group Invariant Solution Corresponding to $\Pi_{1}-c \Pi_{2}$. The linear combination of time and space translation operators gives the travelling wave solution. This type of solution was previously discussed for the flow equation. The wave front type travelling wave solution with constant wave speed $c>0$ gives the invariant as

$$
\theta(y, t)=F(\eta), \quad \text { where } \eta=y+c t,
$$

subjected to

$$
\Pi=\frac{\partial}{\partial t}-c \frac{\partial}{\partial y} .
$$

Using equation (53) into equation (13) gives the secondorder ODE

$$
c \frac{\mathrm{d} F}{\mathrm{~d} \eta}-v_{0} \frac{\mathrm{d} F}{\mathrm{~d} \eta}=\left(\frac{\mathrm{Nr}+\kappa_{*}}{\operatorname{Pr}\left(\rho C_{P}\right)_{*}}\right) \frac{\mathrm{d}^{2} F}{\mathrm{~d} \eta^{2}}+\Phi F,
$$

along with the boundary conditions

$$
\begin{aligned}
F(0) & =1, \\
F(\infty) & =0 .
\end{aligned}
$$

Utilizing the compatibility approach [57], the exact solution of equation (55) is of the form

$$
F(\eta)=\exp \left(-\frac{\left(c-v_{0}\right) \operatorname{Pr}\left(\rho C_{P}\right)_{*}+\sqrt{\left(\rho C_{P}\right)_{*}^{2} \operatorname{Pr}^{2}\left(c-v_{0}\right)^{2}-4\left(\kappa_{*}+\operatorname{Nr}\right) \operatorname{Pr}\left(\rho C_{P}\right)_{*} \Phi}}{2\left(\kappa_{*}+\mathrm{Nr}\right)} \eta\right)
$$

Equation (57) together with equation (53) forms the exact solution of the energy equation:

$$
\theta(y, t)=\exp \left(-\frac{\left(c-v_{0}\right) \operatorname{Pr}\left(\rho C_{P}\right)_{*}+\sqrt{\left(\rho C_{P}\right)_{*}^{2} \operatorname{Pr}^{2}\left(\rho C_{P}\right)^{2}-4\left(\kappa_{*}+\operatorname{Nr}\right) \operatorname{Pr}\left(\rho C_{P}\right)_{*} \Phi}}{2\left(\kappa_{*}+\mathrm{Nr}\right)}(y+\mathrm{ct})\right) .
$$

The above solution satisfies the boundary condition with

$$
\begin{aligned}
& H(t)=\exp \left(-\frac{\left(c-v_{0}\right) \operatorname{Pr}\left(\rho C_{P}\right)_{*}+\sqrt{\left(\rho C_{P}\right)_{*}^{2} \operatorname{Pr}^{2}\left(c-v_{0}\right)^{2}-4\left(\kappa_{*}+\operatorname{Nr}\right) \operatorname{Pr}\left(\rho C_{P}\right)_{*} \Phi}}{2\left(\kappa_{*}+\mathrm{Nr}\right)} c t\right), \\
& G(y)=\exp \left(-\frac{\left(c-v_{0}\right) \operatorname{Pr}\left(\rho C_{P}\right)_{*}+\sqrt{\left(\rho C_{P}\right)_{*}^{2} \operatorname{Pr}^{2}\left(c-v_{0}\right)^{2}-4\left(\kappa_{*}+\mathrm{Nr}\right) \operatorname{Pr}\left(\rho C_{P}\right)_{*} \Phi}}{2\left(\kappa_{*}+\mathrm{Nr}\right)} y\right) .
\end{aligned}
$$

One can notice that above functions depend on the different physical heat transfer parameters which describe the flow and heat transfer behavior.

8.2. Results and Discussion. In this section, the solution (58) is plotted for different heat transfer parameters. The effect of physical parameters on temperature variation of nanofluid is depicted in Figure 4. The effect of increasing nanoparticles concentration within nanofluid is presented in Figure 4(a). The temperature of nanofluid rises with the addition of nanoparticles in the flow stream. It is clear from the figure that the increasing volume concentration of nanoparticles causes to increase in the thermal conductivity which in return decreases the temperature of the fluid. The internal 


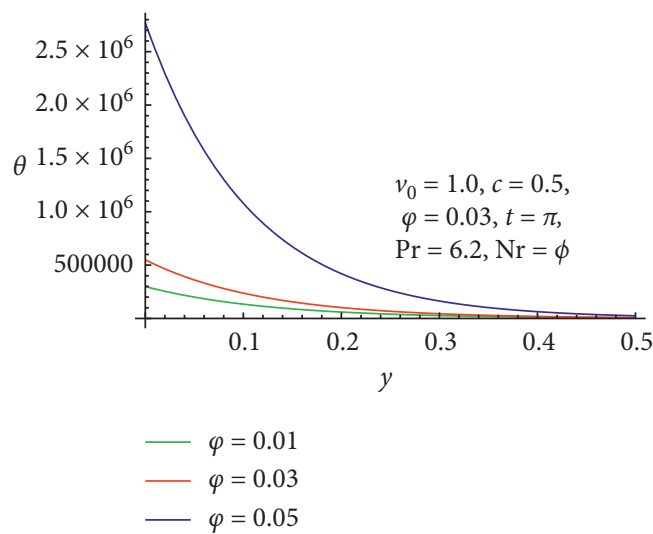

(a)

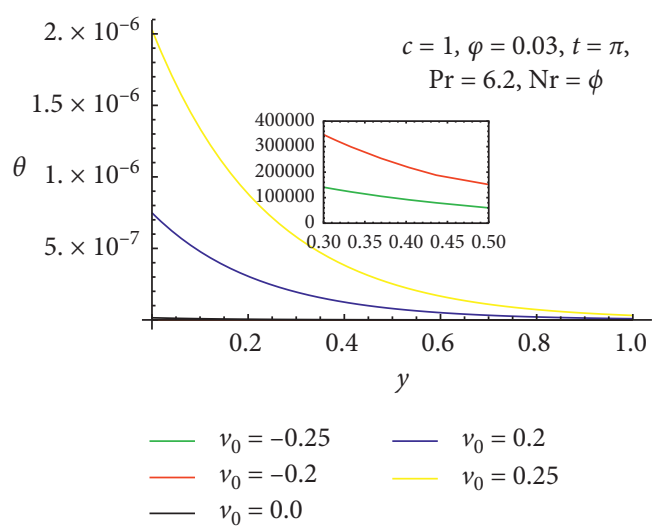

(c)

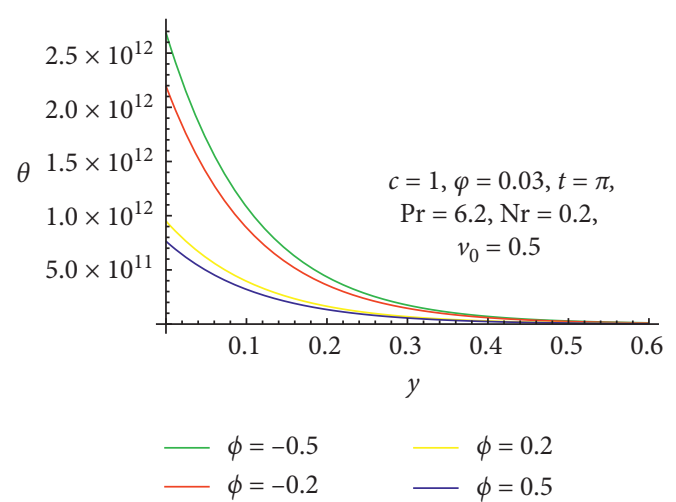

(b)

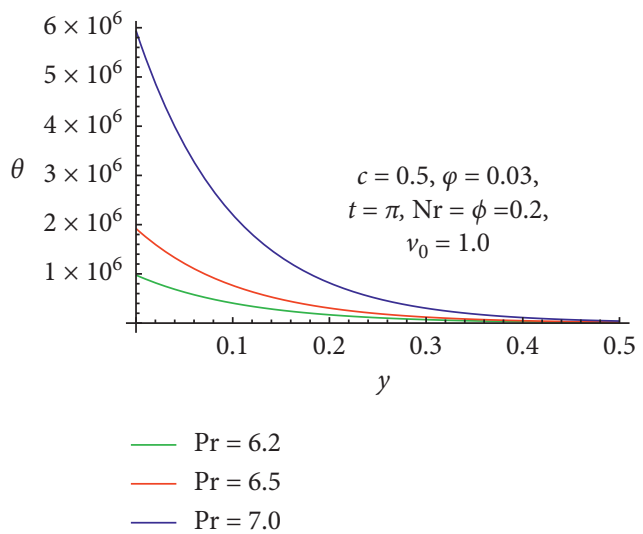

(d)

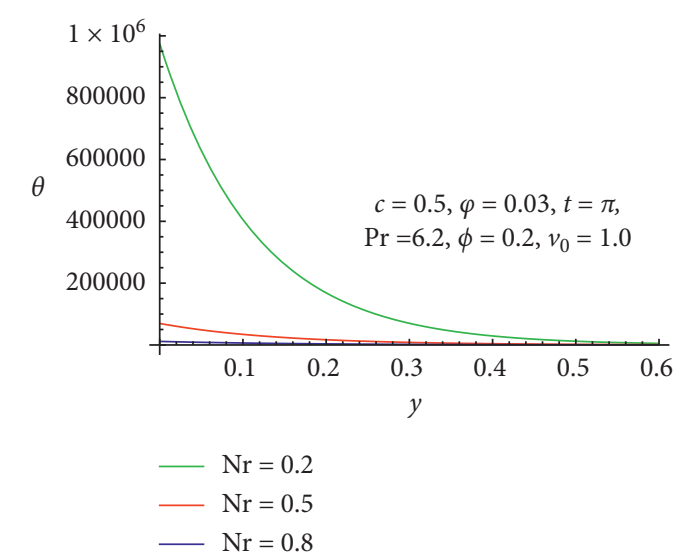

(e)

FIGURE 4: Velocity profile (58) for various values of $\varphi, \Phi, v_{0}$, $\operatorname{Pr}$, and $\mathrm{Nr}$ when $\rho_{s}=8933, \rho_{f}=997.1, C_{P s}=385, C_{P f}=4197, \kappa_{s}=400$, and $\kappa_{f}=0.613$ are fixed.

heat source $\Phi>0$ causes to rise in temperature and thickening of the thermal boundary layer. The internal heat sink $\Phi<0$ reduces the temperature of the fluid which is clear from Figure 4(b). Figure 4(c) elaborates the combined effects of suction $v_{0}>0$ and injection $v_{0}$ on the temperature of the fluid. The temperature is increasing for the increased values of suction and thickening of the thermal boundary layer, and the opposite trend can be observed for injection. In Figure 4(d), the effect of Prandtl number on the temperature profile is presented. Since, the enhancement in Prandtl number corresponds to weaker thermal diffusivity which in return lowers the temperature. The above fact can be observed from Figure 4(d). The thermal boundary layer becomes thin for the increased Prandtl number. It can be seen 
that the temperature at the wall is decreased for larger values of Prandtl number. The effects of radiation parameter on the temperature field are shown in Figure 4(e). It can be observed that the increasing radiation causes to increase in the temperature and thickening of the thermal boundary layer.

\section{Concluding Remarks}

This research aims to extract exact solutions of time-dependent flow and the heat transfer model of power-law nanofluid. To observe the physical behavior of nanofluid for shear thinning and shear thickening fluids, the results are obtained separately for each case. The Lie symmetry method is utilized to get the complete Lie symmetry algebra for the flow and heat transfer model. The symmetry algebra is used to get the reductions of the governing partial differential equation for different values of power-law index $m$. Then, the group invariant solution is presented for the flow and heat transfer model. The effects of physical parameters involving volume fraction of nanoparticles, permeability, suction/injection, and thermal radiation on the flow and heat transfer of the fluid are discussed separately. The exact solution for such type of model in which the non-Newtonian fluids are considered with nanofluids is rare in literature.

The methodology adopted in this article is more precise and used a simple systematic algorithm. The generalized group and compatibility technique aided in finding the exact solution of power-law nanofluid with the heat transfer model. The graphical results are given for different physical parameters to observe the behavior of velocity and temperature distribution for the water-based cu-water nanofluid. The approach used in this article may be extended to other nonlinear problems related to wave mechanics, solid mechanics, and general relativity.

\section{Data Availability}

No data were used to support the findings of this study.

\section{Conflicts of Interest}

The authors declare that they have no conflicts of interest.

\section{References}

[1] E. C. Okonkwo, I. Wole-Osho, I. W. Almanassra et al., "An updated review of nanofluids in various heat transfer devices," Journal of Thermal Analysis and Calorimetry, 2020.

[2] K. V. Wong and O. Leon, "Applications of nanofluids: current and future," Advances in Mechanical Engineering, vol. 2, pp. 1-11, 2010.

[3] W. Yu and H. Xie, "A review on nanofluids: preparation, stability mechanisms, and applications," Journal of Nanomaterials, vol. 2012, Article ID 435873, 17 pages, 2012.

[4] M. Rafati, A. A. Hamidi, and M. Shariati Niaser, "Application of nanofluids in computer cooling systems (heat transfer performance of nanofluids)," Applied Thermal Engineering, vol. 45-46, pp. 9-14, 2012.

[5] J. A. Eastman, S. Choi, S. Li, W. Yu, and L. J. Thompson, "Anomalously increases effective thermal conductvities of ethylene glycol-bases nanofluids containing copper nanoparticles," Applied Physics Letters, vol. 78, no. 6, 2001.

[6] X. Wang, X. Xu, and S. Choi, "Thermal conductivity of nanoparticles-fluid mixture," Journal of Thermophysics and Heat Transfer, vol. 13, p. 474, 1999.

[7] P. Keblinski, S. R. Phillpot, S. Choi, and J. A. Eastman, "Mechanisms of heat flow in suspensions of nano-sized particles (nanofluids)," International Journal of Heat and Mass Transfer, vol. 45, pp. 855-863, 2002.

[8] J. Buongiorno, "Convective transport in nanofluids," ASME Journal of Heat Transfer, vol. 128, pp. 240-250, 2006.

[9] S. K. Das, S. U. S. Choi, and H. E. Patel, "Heat transfer in nanofluids-a review," ASME Heat Transfer Engineering, vol. 27, no. 10, pp. 3-19, 2006.

[10] B. Mahanthesh and J. Mackolil, "Flow of nanoliquid past a vertical plate with novel quadratic thermal radiation and quadratic Boussinesq approximation: sensitivity analysis," International Communications in Heat and Mass Transfer, vol. 120, Article ID 105040, 2021.

[11] S. M. Vanaki, P. Ganesana, and H. A. Mohammed, "Numerical study of convective heat transfer of nanofluids: a review," Renewable and Sustainable Energy Reviews, vol. 54, pp. 1212-1239, 2016.

[12] M. Madhu, B. Mahanthesh, N. S. Shashikumar, S. A. Shehzad, S. U. Khan, and B. J. Gireesha, "Performance of second law in carreau fluid flow by an inclined microchannel with radiative heated convective condition," International Communications in Heat and Mass Transfer, vol. 171, Article ID 104761, 2020.

[13] A. Raees, U. Farooq, M. Hussain et al., "Non-similar mixed convection analysis for magnetic flow of second-grade nanofluid over a vertically stretching sheet," Communications in Theoretical Physics, vol. 73, Article ID 065801, 2021.

[14] A. K. Santra, S. Sen, and N. Chakraborty, "Study of heat transfer due to laminar flow of copper-water nanofluid through two isothermally heated parallel plates," International Journal of Thermal Sciences, vol. 48, no. 2, pp. 391-400, 2009.

[15] W. A. Khana and R. S. R. Golra, "Heat and mass transfer in power-law nanofluids over a nonisothermal stretching wall with convective boundary condition," Journal of Heat Transfer, vol. 134, no. 11, Article ID 112001, 2012.

[16] M. Hojjat, G. S. Etemad, R. Bagheri, and J. Thibault, "Rheological characteristics of non-Newtonian nanofuids: experimental investigation," International Communications in Heat and Mass Transfer, vol. 38, no. 2, pp. 144-148, 2011.

[17] S. Deng, "Thermally fully developed electroosmotic flow of power-law nanofluid in a rectangular microchannel," Micromachines (Basel), vol. 10, no. 6, p. 363, 2019.

[18] S. Deng, Q. An, and M. Li, "The effect of streaming potential and viscous dissipation in the heat transfer characteristics of power-law nanofluid flow in a rectangular microchannel," Micromachines (Basel), vol. 11, no. 4, p. 421, 2020.

[19] A. Aziz and W. Jamshed, "Unsteady MHD slip flow of non Newtonian power-law nanofluid over a moving surface with temperature dependent thermal conductivity," Discrete and Continuous Dynamical Systems-S, vol. 11, no. 4, pp. 617-630, 2018.

[20] M. Khan and W. A. Khana, "MHD boundary layer flow of a power-law nanofluid with new mass flux condition," AIP Advances, vol. 6, Article ID 025211, 2016.

[21] R. Ellahi, S. M. Sait, N. Shehzad, and N. Mobin, "Numerical simulation and mathematical modeling of electro-osmotic Couette-Poiseuille flow of MHD power-law nanofluid with 
entropy generation," Aero/Hydrodynamics and Symmetry, vol. 11, no. 8, Article ID 1038, 2019.

[22] F. Selimefendigil and A. J. Chamkha, "Magnetohydrodynamics mixed convection in a power law nanofluid-filled triangular cavity with an opening using Tiwari and Das nanofluid model," Journal of Thermal Analysis and Calorimetry, vol. 135, pp. 419-436, 2019.

[23] M. W. A. Khan, M. I. Khan, T. Hayat, and A. Alsaedi, "Numerical solution of MHD flow of power law fluid subject to convective boundary conditions and entropy generation," Computer Methods and Programs in Biomedicine, vol. 188, Article ID 105262, 2019.

[24] M. Bisht and V. D. Patil, "Power-law fluid flow in driven enclosures with undulation using MRT-lattice Boltzmann method," Computer and Mathematics with Applications, vol. 79, pp. 100-110, 2020.

[25] W. H. Shen, F. Q. Yang, and Y. P. Zhao, "Unstable crack growth in hydraulic fracturing: the combined effects of pressure and shear stress for a power-law fluid," Engineering Fracture Mechanics, vol. 225, Article ID 106245, 2020.

[26] Q. Xiong, M. V. Bozorg, M. H. Doranehgard, K. Hong, and G. Lorenzini, "A CFD investigation of the effect of nonNewtonian behavior of $\mathrm{Cu}$-water nanofluids on their heat transfer and flow friction characteristics," Journal of Thermal Analysis and Calorimetry, vol. 139, pp. 2601-2621, 2020.

[27] F. Ahmed and M. Iqbal, "Heat transfer analysis of MHD power law nano fluid flow through annular sector duct," Journal of Thermal Science, vol. 29, pp. 169-181, 2020.

[28] A. M. Aziz and A. M. Aly, "MHD boundary layer flow of a power-law nanofluid containing gyrotactic microorganisms over an exponentially stretching surface," Computers, Materials and Continua, vol. 62, pp. 525-549, 2020.

[29] G. Venkatesan and A. S. Reddy, "Insight into the dynamics of blood conveying alumina nanoparticles subject to Lorentz force, viscous dissipation, thermal radiation, Joule heating, and heat source," The European Physical Journal Special Topics, 2021.

[30] M. Ramzan, N. Shaheen, J. D. Chung et al., "Impact of Newtonian heating and Fourier and Fick's laws on a magnetohydrodynamic dusty Casson nanofluid flow with variable heat source/sink over a stretching cylinder," Scientific Reports, vol. 11, Article ID 2357, 2021.

[31] Y. M. Chu., M. Nazeer, M. I. Khan et al., "Combined impacts of heat source/sink, radiative heat flux, temperature dependent thermal conductivity on forced convective Rabinowitsch fluid," International Communications in Heat and Mass Transfer, vol. 120, Article ID 105011, 2021.

[32] P. Agrawal, P. K. Dadheech, R. N. Jat et al., "Magneto Marangoni flow of $\gamma-\mathrm{AL}_{2} \mathrm{O}_{3}$ nanofluids with thermal radiation and heat source/sink effects over a stretching surface embedded in porous medium," Case Studies in Thermal Engineering, vol. 23, Article ID 100802, 2021.

[33] U. Farooq, H. Waqas, M. I. Khan et al., "Thermally radioactive bioconvection flow of Carreau nanofluid with modified Cattaneo-Christov expressions and exponential space-based heat source," Alexendria Engineering Journal, vol. 60, pp. 3073-3086, 2021.

[34] P. Maghsoudi, G. Shahriari, H. Rasam, and S. Sadeghi, "Flow and natural convection heat transfer characteristics of nonNewtonian nanofluid flow bounded by two infinite vertical flat plates in presence of magnetic field and thermal radiation using Galerkin method," Journal of Centeral South University, vol. 26, pp. 1294-1305, 2019.
[35] U. Biswal, S. Chakraverty, and B. K. Ojha, "Natural convection of non-Newtonian nanofluid flow between two vertical parallel plates," International Journal of Numerical Methods for Heat and Fluid Flow, vol. 29, no. 6, pp. 19842008, 2019.

[36] T. Aziz, "Generalized stokes' flow and radiative heat transfer model of a non-Newtonian fluid in a Darcy porous medium subject to Navier's slip conditions on the penetrable porous boundary: group theoretical and compatibility analysis," Applied Mathematics and Computation, vol. 400, Article ID 126048, 2021.

[37] A. Tahiri and K. Mansouri, "Analytical solution by Laplaceritz variational method for non-Newtonian nanofluid inside a circular tube," International Journal of Mechanical Sciences, vol. 135, pp. 596-608, 2018.

[38] A. Aziz and S. Javaid, "Lie symmetry reductions and exact solutions for magnetohydrodynamic flow and heat transfer of third grade nanofluid with thermal radiation," Journal of Thermal Analysis and Calorimetry, vol. 143, pp. 1957-1972, 2021.

[39] T. Aziz, S. Javaid, A. Aziz, and M. A. Sadiq, "Group theoretical analysis for magnetohydrodynamic generalized Stokes' flow and radiative heat transfer model of a non-Newtonian nanofluid with heat generation/absorption," Journal of Thermal Analysis and Calorimetry, vol. 143, pp. 985-1002, 2021.

[40] M. Kezzar, N. Nafir, I. Tabet, and A. Khanetout, "A new analytical investigation of natural convection of non-Newtonian nanofluids flow between two vertical flat plates by the generalized decomposition method (GDM)," Journal of Thermal Engineering, vol. 4, no. 6, pp. 2496-2508, 2018.

[41] T. Aziz, F. M. Mahomed, and A. Aziz, "Group invariant solutions for the unsteady MHD flow of a third grade fluid in a porous medium," International Journal of Non-linear Mechanics, vol. 47, pp. 792-798, 2012.

[42] T. Aziz, A. Fatima, A. Aziz, and F. M. Mahomed, "Group theoretical analysis and invariant solutions for unsteady flow of a fourth-grade fluid over an infinite plate undergoing impulsive motion in a Darcy porous medium," Zeitschrift für Naturforschung A, vol. 70, pp. 483-497, 2015.

[43] T. Aziz, A. Aziz, and C. M. Khalique, "Exact solutions for stoke's flow of a non-Newtonian nanofluid model: a lie similarity approach," Zeitschrift für Naturforschung A, vol. 71, 2016.

[44] T. Amtout, H. Biyadi, M. Er-Riani, and M. E. Jarroudi, "Lie group analysis for heat transfer in flow of second grade fluid," International Journal of Dynamical Systems and Differential Equations, vol. 9, no. 164, 2019.

[45] G. Magalakwe, M. L. Lekoko, K. Modise, and C. M. Khalique, "Lie group analysis for MHD squeezing flow of viscous fluid saturated in porous media," Alexandria Engineering Journal, vol. 58, pp. 1001-1010, 2019.

[46] A. Hussain, S. Bano, I. Khan, D. Baleanu, and K. S. Nisar, "Lie group analysis, explicit solutions and conservation laws of a spatially two-dimensional Burgers-Huxley equation," Symmetry, vol. 12, no. 170, 2020.

[47] K. Bibi, "Particular solutions of ordinary differential equations using discrete symmetry groups," Symmetry, vol. 12, 2020.

[48] R. J. Tiwari and M. K. Das, "Heat transfer augmentation in a two-sided lid-driven differentially heated square cavity utilizing nanofluids," International Journal of Heat and Mass Transfer, vol. 50, pp. 2002-2018, 2007.

[49] F. M. White, Fluid Mechanics, WCB/McGraw Hill, New York, NY, USA, 1999. 
[50] R. L. Hamilton and O. K. Crosser, "Thermal conductivity of heterogeneous two component systems," Industrial and Engineering Chemistry Fundamentals, vol. 1, no. 13, pp. 27-40, 1962.

[51] N. Bhaskar, P. T. Reddy, and P. Sreenivasulu, "Influence of variable thermal conductivity on MHD boundary layer slip flow of ethylene-glycol based $\mathrm{Cu}$ nanofluids over a stretching sheet with convective boundary condition," International Journal of Engineering Mathematics, vol. 2014, 2014.

[52] R. N. R. Arunachalam, "Forced convection in liquid metals with variable thermal conductivity and capacity," Acta Mechanica, vol. 31, no. 25-31, 1978.

[53] J. Maxwell, A Treatise on Electricity and Magnesium, Clarendon Press, Oxford, UK, 2nd edition, 1881.

[54] R. B. Bird, W. E. Stewar, and E. N. Lightfoot, Transport Phenomena, John Wiley \& Sons, New York, NY, USA, 1960.

[55] F. M. Sparrow and R. D. Cess, Radiation Heat Transfer, Vol. 231, Hemisphere Publisher, London, UK, 1995.

[56] M. Q. Brewster, Thermal Radiative Transfer and Proprties, John Wiley and Sons, New York, NY, USA, 1992.

[57] T. Aziz, F. M. Mahomed, and D. P. Mason, "A unified compatibility method for exact solutions of nonlinear flow models of Newtonian and non- Newtonian fluids," International Journal of Non-linear Mechanics, vol. 78, pp. 142-155, 2016. 